\title{
Rate versus rhythm control and outcomes in patients with atrial fibrillation and chronic kidney disease: Data from the GUSTO-III Trial
}

\author{
Eric S. Williams ${ }^{1}$, Vivian P. Thompson ${ }^{2}$, Karen E. Chiswell ${ }^{2}$, John H. Alexander ${ }^{2}$, \\ Harvey D. White ${ }^{3}$, E. Magnus Ohman ${ }^{2}$, Sana M. Al-Khatib ${ }^{2}$ \\ ${ }^{1}$ Division of Cardiology, University of Washington Medical Center, Seattle, WA, USA \\ ${ }^{2}$ Division of Cardiology and Duke Clinical Research Institute, Duke University, \\ Medical Center, Durham, NC, USA \\ ${ }^{3}$ Green Lane Cardiovascular Service, Auckland City Hospital, Auckland, New Zealand
}

\begin{abstract}
Background: Atrial fibrillation (AF) and chronic kidney disease (CKD) have both been shown to portend worse outcomes after acute myocardial infarction (MI); however, the benefit of a rhythm control strategy in patients with CKD post-MI is unclear.

Methods: We prospectively studied 985 patients with new-onset AF post-MI in the GUSTO-III trial, of whom 413 (42\%) had CKD (creatinine clearance $<60 \mathrm{~mL} / \mathrm{min}$ ). A rhythm control strategy, defined as the use of an antiarrhythmic medication and/or electrical cardioversion, was used in 346 (35\%) of patients.

Results: A rhythm control strategy was used in 34\% of patients with CKD and $36 \%$ of patients with no CKD. At hospital discharge, sinus rhythm was present in 487 (76\%) of patients treated with a rate control strategy, $v$ s. $276(80 \%)$ in those treated with rhythm control $(p=0.20)$. $C K D$ was associated with a lower odds of sinus rhythm at discharge (unadjusted OR 0.56, 95\% CI $0.38-0.84, p<0.001)$. However, in multivariable analyses, treatment with a rhythm control strategy was not associated with discharge rhythm (HR 1.068, 95\% CI 0.69-1.66, $p=0.77$ ), 30-day mortality (HR 0.78, 95\% CI 0.54-1.12, $p=0.18$ ) or mortality from day 30 to 1 year (HR 1.00, 95\% CI 0.59-1.69, $p=0.99$ ). CKD status did not significantly impact the relationship between rhythm control and outcomes.

Conclusions: Treatment with a rhythm or rate control strategy does not significantly impact short-term or long-term mortality in patients with post-MI AF, regardless of kidney disease status. Future studies to investigate the optimal management of $A F$ in CKD patients are needed. (Cardiol J 2013; 20, 4: 439-446)
\end{abstract}

Key words: atrial fibrillation, chronic kidney disease, antiarrhythmic medications, myocardial infarction

Address for correspondence: Sana M. Al-Khatib, MD, MHS, Duke Clinical Research Institute, 2400 Pratt Street, Durham, NC 27705, USA, tel: +001 (919) 668-6849, fax: +001 (919) 681-9260, e-mail: alkha001@mc.duke.edu 


\section{Introduction}

Atrial fibrillation (AF) is twice as prevalent among patients with chronic kidney disease (CKD) and over 10 times more prevalent in patients on dialysis than in the general population [1, 2]. In the Chronic Renal Insufficiency Cohort of 3,267 adult participants, nearly 1 in 5 participants had evidence of AF; age, female gender, and a history of cardiovascular disease were the major predictors of AF in the study. Also, among 956 outpatients with coronary artery disease in the Heart and Soul Study, kidney dysfunction was independently associated with prevalent AF [3]. Although each of these groups of patients is at high risk for cardiovascular mortality and morbidity, little is known about the impact of AF treatment strategy on clinical outcomes in patients with kidney disease. As such, the optimal management of AF in this population is unknown.

$\mathrm{AF}$ in the setting of acute myocardial infarction (MI) is associated with worse outcomes [4]. Although antiarrhythmic medications with or without electrical cardioversion may restore sinus rhythm (SR) in these patients, their ability to improve post-MI survival is unclear. In the Valsartan in Acute Myocardial Infarction (VALIANT) Trial, antiarrhythmic use was associated with excess short-term mortality in the peri-MI period but not long-term mortality [5]. Results from the Global Use of Strategies to Open Occluded Coronary Arteries III (GUSTO-III) AF substudy suggested a trend toward lower mortality with the use of sotalol and procainamide in post-MI patients with $\mathrm{AF}$ [6]. The effect of CKD on these outcomes is unknown. This is an issue of particular clinical significance, because CKD itself is a risk factor for increased mortality after acute MI [7, 8].

Using prospective data from the GUSTO-III trial, we conducted an analysis to investigate the use of a rhythm control strategy in patients with CKD and post-MI AF and to study its impact on the maintenance of SR, 30-day and 1-year mortality.

\section{Methods}

\section{Study population}

The GUSTO-III trial was an international study of over 15,000 patients treated for acute MI with ST-segment elevation or new left bundle-branch block and randomized to alteplase or reteplase therapy between October 13, 1995 and January 13, 1997 [9]. A cohort of 1,138 subjects who developed $\mathrm{AF}$ during the study period was enrolled into a prospective substudy of post-MI AF management [6].
Patients were treated either with a rate control only strategy using atrioventricular nodal blocking agents or a rhythm control strategy using antiarrhythmic medications and/or electrical cardioversion. Treatment decisions were made by the primary medical team and were not randomized, but this pre-specified observational analysis investigated outcomes including the maintenance of SR, and mortality at 30 days and 1 year post-MI. Patients were excluded from this analysis if they had longstanding persistent or permanent $\mathrm{AF}$ at study entry.

The details of patient enrollment and data collection have been previously described [9]. The Duke University Medical Center Institutional Review Board approved the study protocol, and all patients provided informed written consent for the collection of clinical data and blood samples before participation in the GUSTO-III trial.

\section{Data collection}

This was a prospective cohort analysis of outcomes in the GUSTO-III AF substudy, stratified by the presence or absence of CKD (defined as a calculated creatinine clearance below $60 \mathrm{~mL} / \mathrm{min} / 1.73 \mathrm{~m}^{2}$ ) at the time of enrollment. The primary outcome measure was 1-year survival post AF onset. Secondary outcomes included 30-day survival post $\mathrm{AF}$ onset and the presence of $\mathrm{SR}$ at the time of hospital discharge or last stable rhythm prior to death. The goal of the proposed analysis was to investigate the use of a rate vs. rhythm control strategy in patients with post-MI AF and to study the impact of $\mathrm{CKD}$ and treatment strategy on the maintenance of SR, 30-day and 1-year mortality. Data collected as part of the substudy included the use of ventricular rate controlling agents (beta-blockers, calcium antagonists, or digitalis), the use of any antiarrhythmic agent (procainamide, quinidine, disopyramide, encainide, flecainide, propafenone, sotalol and amiodarone) or electrical cardioversion for rhythm control, the use of antiplatelet and anticoagulant medications at hospital discharge, and the cardiac rhythm at hospital discharge or the last stable rhythm recorded before deterioration to in-hospital death.

\section{Statistical analysis}

Baseline variables were compared between groups using the Wilcoxon rank-sum test for continuous variables and the Pearson $\chi^{2}$ or Exact test for categorical variables. The product-limit method was used to model the event-free survival of subjects, and survival curves were compared using the log-rank test. Factors associated with survival time after the onset of AF were assessed in univariate and multivariable Cox proportional hazards 
Table 1. Baseline characteristics of the cohort by treatment strategy and by chronic kidney disease (CKD) status.

\begin{tabular}{|c|c|c|c|c|c|c|}
\hline & \multicolumn{3}{|c|}{ Rate vs. rhythm control } & \multicolumn{3}{|c|}{ CKD vs. non-CKD } \\
\hline & $\begin{array}{c}\text { Rate } \\
(n=639)\end{array}$ & $\begin{array}{l}\text { Rhythm } \\
(\mathrm{n}=346)\end{array}$ & $\mathbf{P}$ & $\begin{array}{c}\text { CKD } \\
(n=413)\end{array}$ & $\begin{array}{l}\text { Non-CKD } \\
(\mathrm{n}=559)\end{array}$ & $\mathbf{P}$ \\
\hline Age [years] ${ }^{*}$ & $69.9 \pm 10.5$ & $68.4 \pm 10.9$ & 0.054 & $75.9 \pm 7.6$ & $64.6 \pm 9.9$ & $<0.001$ \\
\hline White race & $95.4 \%$ & $96.0 \%$ & 0.752 & $97.3 \%$ & $94.6 \%$ & 0.022 \\
\hline Women & $34.1 \%$ & $34.1 \%$ & 0.997 & $50.6 \%$ & $21.6 \%$ & $<0.001$ \\
\hline Hypertension & $49.0 \%$ & $46.5 \%$ & 0.462 & $51.1 \%$ & $46.5 \%$ & 0.158 \\
\hline Hyperlipidemia & $30.4 \%$ & $35.5 \%$ & 0.108 & $27.0 \%$ & $35.9 \%$ & 0.003 \\
\hline Prior coronary bypass & $3.1 \%$ & $3.2 \%$ & 0.966 & $2.7 \%$ & $3.6 \%$ & 0.423 \\
\hline $\begin{array}{l}\text { Prior coronary } \\
\text { angioplasty/stent }\end{array}$ & $5.3 \%$ & $4.9 \%$ & 0.783 & $4.4 \%$ & $5.7 \%$ & 0.341 \\
\hline Prior MI & $22.7 \%$ & $20.5 \%$ & 0.432 & $24.5 \%$ & $20.0 \%$ & 0.100 \\
\hline Tobacco history & $64.7 \%$ & $64.5 \%$ & 0.400 & $55.7 \%$ & $71.2 \%$ & $<0.001$ \\
\hline Cerebrovascular disease & $3.1 \%$ & $2.9 \%$ & 0.831 & $4.1 \%$ & $2.3 \%$ & 0.109 \\
\hline Systolic blood pressure & $118.1 \pm 27.5$ & $117.0 \pm 24.4$ & 0.486 & $118.0 \pm 27.1$ & $117.9 \pm 25.6$ & 0.890 \\
\hline Diastolic blood pressure & $72.1 \pm 36.6$ & $69.6 \pm 15.0$ & 0.376 & $70.9 \pm 35.8$ & $71.7 \pm 26.2$ & 0.427 \\
\hline Heart rate & $107.4 \pm 35.5$ & $122.6 \pm 33.1$ & $<0.001$ & $111.7 \pm 34.6$ & $114.0 \pm 35.9$ & 0.289 \\
\hline Killip class: & & & 0.048 & & & $<0.001$ \\
\hline 1 & $75.8 \%$ & $75.7 \%$ & & $68.8 \%$ & $80.9 \%$ & \\
\hline II & $20.7 \%$ & $20.6 \%$ & & $26.8 \%$ & $16.2 \%$ & \\
\hline III & $1.3 \%$ & $3.2 \%$ & & $2.2 \%$ & $1.8 \%$ & \\
\hline IV & $2.2 \%$ & $0.6 \%$ & & $2.2 \%$ & $1.1 \%$ & \\
\hline MI location: & & & 0.009 & & & 0.425 \\
\hline Anterior & $43.8 \%$ & $54.6 \%$ & & $49.4 \%$ & $46.3 \%$ & \\
\hline Inferior & $51.6 \%$ & $41.6 \%$ & & $45.8 \%$ & $50.1 \%$ & \\
\hline Other & $4.2 \%$ & $3.5 \%$ & & $4.6 \%$ & $3.2 \%$ & \\
\hline LVEF $[\%]^{*}$ & $45.7 \pm 14.3$ & $45.6 \pm 14.2$ & 0.677 & $45.1 \pm 16.4$ & $45.9 \pm 13.0$ & 0.691 \\
\hline Creatinine $[\mathrm{mg} / \mathrm{dL}]^{*}$ & $1.02 \pm 0.30$ & $1.01 \pm 0.30$ & 0.376 & $1.17 \pm 0.35$ & $0.91 \pm 0.21$ & $<0.001$ \\
\hline $\begin{array}{l}\text { Creatinine clearance } \\
{[\mathrm{mL} / \mathrm{min}]^{*}}\end{array}$ & $67.6 \pm 28.1$ & $70.8 \pm 30.2$ & 0.154 & $44.9 \pm 10.6$ & $86.3 \pm 25.3$ & $<0.001$ \\
\hline
\end{tabular}

${ }^{*}$ Continuous variables given as mean $\pm \mathrm{SD} ; \mathrm{MI}$ - myocardial infarction; LVEF — left ventricular ejection fraction

models. In addition to CKD and treatment strategy, covariates previously known to be associated with mortality in the GUSTO trial were included in the models $[9,10]$. Additionally, factors associated with maintenance of SR at hospital discharge were modeled using multivariable logistic regression. Candidate variables selected by the study investigators were considered in multivariable stepwise regression models, and a 2 -tailed $p$ value of $<0.05$ was considered statistically significant. Analyses were performed using SAS, version 9.2, software (SAS Institute, Cary, NC).

\section{Results}

\section{Population characteristics}

Of the 1,138 patients enrolled in the GUSTO-III AF substudy, 153 were excluded due to a history of longstanding persistent or permanent AF. Thus, 985 patients met our inclusion criteria and were represented in this analysis. Of these patients, $639(65 \%)$ were treated with a rate control only strategy, and $346(35 \%)$ were treated with a rhythm control strategy during the index hospitalization. The baseline characteristics of the cohort are shown in Table 1. Subjects who received rhythm control on average had higher heart rates at enrollment and were more likely to present with an anterior MI than those who received a rate control strategy; otherwise, no significant differences in demographic characteristics or baseline medical conditions were noted between the two groups. Additionally, there was no significant difference in the percentage of patients managed with a rhythm control strategy in the CKD vs. non-CKD groups (34\% vs. $36 \%)$. Subjects with CKD had a mean 
Table 2. Summary of antiarrhythmic management in the rhythm control group.

\begin{tabular}{lcccc}
\hline & Total* $(\mathbf{n}=\mathbf{3 4 6})$ & CKD** $(\mathbf{n}=\mathbf{1 4 2})$ & Non-CKD** $(\mathbf{n}=\mathbf{2 0 1})$ & $\mathbf{P}$ \\
\hline Amiodarone & $45.4 \%(157)$ & $50.0 \%(71)$ & $41.8 \%(84)$ & 0.362 \\
Procainamide & $24.6 \%(85)$ & $18.3 \%(26)$ & $28.9 \%(58)$ & 0.025 \\
Sotalol & $11.8 \%(41)$ & $12.0 \%(17)$ & $11.9 \%(24)$ & 0.892 \\
Propafenone & $5.8 \%(20)$ & $5.6 \%(8)$ & $6.0 \%(12)$ & 0.820 \\
Quinidine & $5.2 \%(18)$ & $5.6 \%(8)$ & $5.0 \%(10)$ & 0.866 \\
Disopyramide & $0.9 \%(3)$ & $1.4 \%(2)$ & $0.5 \%(1)$ & 0.578 \\
Flecainide & $0.6 \%(2)$ & $0.7 \%(1)$ & $0.5 \%(1)$ & 1.000 \\
\hline
\end{tabular}

*Some subjects in rhythm control group received electrical cardioversion without antiarrhythmic medications; ${ }^{* *}$ creatinine clearance data missing in 3 subjects; CKD - chronic kidney disease

Table 3. Antithrombotic use in the cohort at hospital discharge.

\begin{tabular}{|c|c|c|c|c|c|c|}
\hline & \multicolumn{3}{|c|}{ Rate vs. rhythm control } & \multicolumn{3}{|c|}{ CKD vs. non-CKD } \\
\hline & $\begin{array}{c}\text { Rate } \\
(\mathrm{n}=639)\end{array}$ & $\begin{array}{l}\text { Rhythm } \\
(\mathrm{n}=346)\end{array}$ & $\mathbf{P}$ & $\begin{array}{c}\text { CKD } \\
(n=413)\end{array}$ & $\begin{array}{l}\text { Non-CKD } \\
(\mathrm{n}=559)\end{array}$ & $\mathbf{P}$ \\
\hline Aspirin & $76.4 \%$ & $76.5 \%$ & 0.982 & $71.5 \%$ & $79.5 \%$ & 0.006 \\
\hline Warfarin & $16.0 \%$ & $22.8 \%$ & 0.008 & $19.4 \%$ & $17.7 \%$ & 0.509 \\
\hline Heparin (subcutaneous) & $1.4 \%$ & $2.3 \%$ & 0.299 & $1.0 \%$ & $2.3 \%$ & 0.111 \\
\hline $\begin{array}{l}\text { Other antiplatelet (thienopyridine, } \\
\text { dipyridamole) }\end{array}$ & $11.1 \%$ & $9.0 \%$ & 0.290 & $7.0 \%$ & $12.7 \%$ & 0.004 \\
\hline
\end{tabular}

CKD - chronic kidney disease

creatinine clearance of $45 \mathrm{~mL} / \mathrm{min}$, were older, more likely to be white and female, tended to have slightly worse Killip class, and were less likely to have a history of hyperlipidemia and smoking than subjects without CKD.

\section{Management strategy}

Table 2 summarizes the details of the treatment strategy in the rhythm control group. In this group, 30\% (103) received electrical cardioversion, $55 \%$ of whom had a primary cardioversion strategy, as opposed to antiarrhythmic medication use followed by cardioversion. Amiodarone and procainamide were the principal antiarrhythmic agents used in the rhythm control cohort, used in $70 \%$ of subjects. Procainamide was used less frequently in CKD patients than in non-CKD patients $(p=0.025)$; no difference was seen with the use of other antiarrhythmic agents. Class IC antiarrhythmics were used infrequently in the cohort. Specifically, flecainide was only used in 2 subjects, and encainide was not used at all, very likely due to their known association with increased mortality in the post-MI setting.

Table 3 shows the use of antiplatelet and anticoagulant medications at hospital discharge, in each subgroup. Notably, patients in the rhythm control group were more likely to receive warfarin at discharge than those in the rate control group; the rates of use of other antithrombotics in the two groups were similar. In addition, patients with CKD were less likely to receive aspirin and other antiplatelet medication than those without CKD; warfarin and subcutaneous heparin, however, were used similarly in CKD and non-CKD patients.

\section{Survival analysis}

At follow-up, there were a total of 154 (15.6\%) deaths within 30 days and $222(22.5 \%)$ deaths at 1 year. Kaplan-Meier curves for survival from the time of AF onset up to 1 year are displayed in the Figure 1. Observed survival differences were largest between those with and without CKD (log-rank p-value $<0.001$ ), with non-significant differences between rate and rhythm control strategies (log-rank p-value $=0.109)$. Table 4 shows that in univariate analysis, there was a statistically significant association between CKD and increased mortality at 30 days (unadjusted HR 2.4, 95\% CI 1.7-3.3, p < 0.001) and from day 30 to 1 year (unadjusted HR $1.7,95 \%$ CI $1.1-2.8$, $\mathrm{p}=0.027)$. However, in multivariable analyses, the 


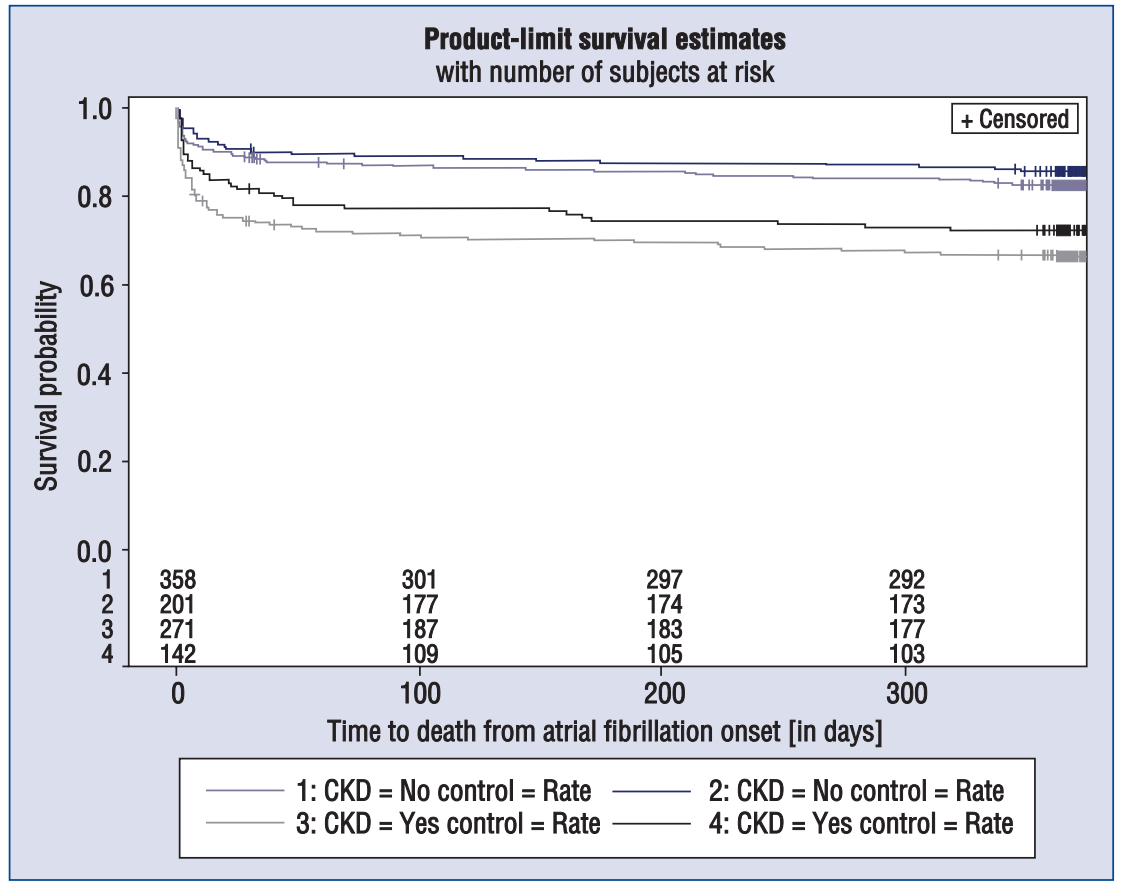

Figure 1. Unadjusted survival curves for all-cause mortality up to 1 year; CKD — chronic kidney disease.

Table 4. Proportional hazards models for all-cause mortality.

\begin{tabular}{|c|c|c|c|c|c|c|}
\hline \multirow[t]{2}{*}{ Analysis } & \multicolumn{3}{|c|}{ Atrial fibrillation onset to Day $30 *$} & \multicolumn{3}{|c|}{ Day 30 to 1 year** } \\
\hline & $\begin{array}{c}\text { Hazard } \\
\text { ratio }\end{array}$ & $\begin{array}{l}95 \% \text { confidence } \\
\text { interval }\end{array}$ & $\mathbf{P}$ & $\begin{array}{l}\text { Hazard } \\
\text { ratio }\end{array}$ & $\begin{array}{l}95 \% \text { confidence } \\
\text { interval }\end{array}$ & $\mathbf{P}$ \\
\hline \multicolumn{7}{|l|}{ Univariate: } \\
\hline Kidney disease & 2.40 & $1.73-3.34$ & $<0.001$ & 1.72 & $1.07-2.76$ & 0.027 \\
\hline Strategy (rhythm vs. rate) & 0.73 & $0.52-1.04$ & 0.073 & 0.94 & $0.57-1.55$ & 0.809 \\
\hline \multicolumn{7}{|l|}{ Multivariable: } \\
\hline Kidney disease & 1.42 & $0.92-2.20$ & 0.118 & 0.90 & $0.49-1.65$ & 0.735 \\
\hline Strategy (rhythm vs. rate) & 0.78 & $0.54-1.12$ & 0.183 & 1.00 & $0.59-1.69$ & 0.992 \\
\hline
\end{tabular}

*Multivariable model adjusted for age, weight, entry blood pressure, Killip class at entry, smoking status, prior coronary artery bypass grafting, hypertension, cerebrovascular disease, diabetes mellitus, entry pulse, previous myocardial infarction and time from symptom onset to atrial fibrillation. Interaction between kidney disease and strategy was found not statistically significant ( $p=0.8899)$

**Multivariable model adjusted for age, weight, entry blood pressure, Killip class at entry, smoking status, prior coronary artery bypass grafting, hypertension, cerebrovascular disease, diabetes mellitus, entry pulse, previous myocardial infarction, prior percutaneous transluminal coronary angioplasty, previous angina, myocardial infarction location, race, gender and US enrollment. Interaction between kidney disease and strategy was found not statistically significant $(p=0.3815)$

association between CKD and mortality became non-significant. In addition, regardless of kidney disease status, treatment with a rate vs. rhythm control strategy was not significantly associated with mortality ( $p$ value for interaction between CKD and treatment strategy $=0.89$ for 30 -day and 0.38 for 1 -year mortality).

\section{Efficacy of rhythm control}

Sinus rhythm was present at hospital discharge in $487(76 \%)$ of patients treated with a rate control strategy, vs. $276(80 \%)$ in those treated with rhythm control $(p=0.20)$. In univariate analysis, CKD was associated with a lower odds of SR at discharge (unadjusted OR 0.56, 95\% CI 0.38-0.84, $\mathrm{p}<0.001$ ), as shown in Table 5. In multivariable analysis that adjusted for potential confounders, however, treatment with a rhythm control strategy was not associated with discharge rhythm (HR 1.068, 95\% CI 0.69-1.66, p = 0.77), 30-day mortality (HR $0.78,95 \%$ CI $0.54-1.12, \mathrm{p}=0.18$ ) or mortality from day 30 to 1 year (HR $1.00,95 \%$ CI 
Table 5. Odds ratios for sinus rhythm at discharge.

\begin{tabular}{lccc}
\hline Analysis & Odds ratio & 95\% confidence interval & P \\
\hline Univariate: & & & $<0.001$ \\
$\quad$ Kidney disease & 0.56 & $0.375-0.838$ & 0.447 \\
$\quad$ Strategy (rhythm vs. rate) & 1.18 & $0.772-1.792$ & 0.4553 \\
Multivariable*: & & & 0.7706 \\
$\quad$ Kidney disease & 0.829 & $0.506-1.357$ & $0.687-1.661$ \\
$\quad$ Strategy (rhythm vs. rate) & 1.068 & & \\
\hline
\end{tabular}

* Multivariable analysis adjusted for age, diastolic blood pressure (pressure $>85$ ), Killip class at entry. Interaction term between kidney disease and strategy was found not statistically significant $(p=0.3023)$

Table 6. Sensitivity analysis for all-cause mortality in moderate to severe chronic kidney disease.

\begin{tabular}{|c|c|c|c|c|c|c|}
\hline & \multicolumn{3}{|c|}{ Atrial fibrillation onset to Day $30 *$} & \multicolumn{3}{|c|}{ Day 30 to 1 year** } \\
\hline & $\begin{array}{c}\text { Hazard } \\
\text { ratio }\end{array}$ & $\begin{array}{c}95 \% \text { confidence } \\
\text { interval }\end{array}$ & $\mathbf{P}$ & $\begin{array}{c}\text { Hazard } \\
\text { ratio }\end{array}$ & $\begin{array}{c}\text { 95\% confidence } \\
\text { interval }\end{array}$ & $\mathbf{P}$ \\
\hline \multicolumn{7}{|l|}{ Multivariable analysis } \\
\hline Creatinine clearance $<45$ & 1.93 & $1.28-2.92$ & 0.002 & 1.93 & $1.02-3.66$ & 0.045 \\
\hline Strategy (rhythm vs. rate) & 0.76 & $0.53-1.09$ & 0.136 & 0.96 & $0.57-1.63$ & 0.890 \\
\hline
\end{tabular}

* Model adjusted for age, weight, entry blood pressure, Killip class at entry, smoking status, prior coronary artery bypass grafting, hypertension, cerebrovascular disease, diabetes mellitus, entry pulse, previous myocardial infarction and time from symptom onset to atrial fibrillation. Interaction between moderate to severe chronic kidney disease and strategy was found not statistically significant ( $p=0.3974$ ) **Model adjusted for age, weight, entry blood pressure, Killip class at entry, smoking status, prior coronary artery bypass grafting, hypertension, cerebrovascular disease, diabetes mellitus, entry pulse, previous myocardial infarction, prior percutaneous transluminal coronary angioplasty, previous angina, myocardial infarction location, race, gender and US enrollment. Interaction between moderate to severe chronic kidney disease and strategy was found not statistically significant $(p=0.3979)$

Table 7. Sensitivity analysis for sinus rhythm at hospital discharge in moderate to severe chronic kidney disease.

\begin{tabular}{lccc}
\hline Multivariable analysis* & Odds ratio & 95\% confidence interval & P \\
\hline Creatinine clearance $<45$ & 0.90 & $0.53-1.55$ & 0.711 \\
Strategy (rhythm vs. rate) & 1.06 & $0.68-1.65$ & 0.792 \\
\hline
\end{tabular}

* Model adjusted for age, diastolic blood pressure (pressure $>85$ ), Killip class at entry. Interaction between moderate to severe chronic kidney disease and strategy was found statistically significant $(p=0.0145)$

0.59-1.69, $\mathrm{p}=0.99$ ) (Tables 4, 5). Additionally, the lack of association between treatment strategy and discharge rhythm was not significantly different in CKD patients ( $\mathrm{p}$ value for interaction between CKD and treatment strategy $=0.30$ ).

\section{Sensitivity analysis}

Regression analyses were repeated to examine the effect of moderate to severe CKD, defined as a creatinine clearance $<45 \mathrm{~mL} / \mathrm{min}$, on clinical outcomes (Tables 6,7 ). In multivariable analysis, there was an almost 2-fold increase in mortality associated with moderate to severe CKD at 30 days (HR 1.93, 95\% CI 1.28-2.92, $\mathrm{p}=0.002$ ) and from day 30 to 1 year (HR 1.93, 95\% CI 1.02-3.66, $\mathrm{p}=0.04)$. There was no significant association between treatment with a rate vs. rhythm control strategy and mortality in the subgroup with creati- nine clearance $<45 \mathrm{~mL} / \mathrm{min}$ ( $\mathrm{p}$ value for interaction between moderate to severe CKD and treatment strategy $=0.40$ both for 30-day and for 1-year mortality). There was a difference, however, in the effect of treatment strategy on discharge rhythm in this subgroup. While in the overall cohort there was no association between treatment strategy and discharge rhythm, in the patients with moderate to severe CKD, there was a lower probability of SR at discharge with a rhythm control strategy (adjusted OR 0.43, 95\% CI 0.19-0.99, p value for interaction between moderate to severe CKD and treatment strategy $=0.01$ ).

\section{Discussion}

In this prospective cohort analysis of patients with AF complicating acute MI, we examined the 
impact of both CKD and treatment with a primary rate or rhythm control strategy on clinical outcomes. Over a third of subjects in the GUSTO-III substudy who developed AF were treated with a rhythm control strategy, which primarily consisted of antiarrhythmic agents such as amiodarone or procainamide. Thirty percent of patients received electrical cardioversion, either alone or with adjunctive antiarrhythmic therapy. There was no significant difference in the use of rhythm control between the CKD and non-CKD arms. In our study, treatment with a rate or rhythm control strategy was not significantly associated with 30-day and 1 -year outcomes in multivariable analyses. In unadjusted analyses, subjects with CKD had increased mortality and were less likely to maintain SR at discharge; in multivariable analysis there was no significant association between CKD and either outcome, regardless of treatment strategy. A sensitivity analysis was performed to investigate the outcomes of subjects with moderate to severe $\mathrm{CKD}$, defined as creatinine clearance $<45 \mathrm{~mL} / \mathrm{min}$, which found a lower likelihood of maintaining SR at discharge with a rhythm control strategy in multivariable analysis.

While AF diagnosed in the setting of acute MI has been associated with adverse outcomes [4, 10-13], the optimal treatment strategy of AF in this setting is unclear, with some studies suggesting an increased harm with antiarrhythmic therapy [5], and others either neutral or suggesting a possible benefit $[6,14]$. It is known that the development of post-MI AF is associated with other factors that independently portend a more complicated clinical course, such as older age, left ventricular dysfunction, and worsening heart failure [4, 15]. To the extent that $\mathrm{AF}$ is a marker of these adverse prognostic factors, treatment directed at controlling $\mathrm{AF}$ per se, may not necessarily improve clinical outcomes. In addition, the risk of pro-arrhythmia related to antiarrhythmic medication use has to be weighed against any potential benefit of restoring and maintaining SR. In the present study, there was no significant association between $\mathrm{AF}$ treatment strategy and either short- or long-term mortality. This suggests that, while an aggressive strategy of rhythm control may not be indicated in the routine management of all patients with post-MI AF, it is certainly reasonable to consider in the short-term management of select patients in the post-MI setting, especially in patients who are symptomatic despite adequate rate control [6].

CKD is both a risk factor for $\mathrm{AF}[2,16,17]$ and an independent predictor of adverse outcomes after acute MI $[8,18-20]$. The risks of antiarrhythmic therapy may be magnified in patients with CKD, owing to the need for renal dose adjustment of certain antiarrhythmic agents [21], as well as to the co-morbidity that often accompanies CKD and that may promote pro-arrhythmia. The development of fibrosis and left ventricular hypertrophy associated with kidney disease is hypothesized to predispose CKD patients to cardiac arrhythmia and sudden death by its effects on the QT interval and QT dispersion [22, 23]. As a result, evidence from clinical trials on rate and rhythm control strategies in AF may not be generalizable to patients with impaired renal function, so data are limited in this regard. This is the first study we are aware of that addresses the safety and efficacy of rate vs. rhythm control of AF in CKD patients. Notably, despite high post-MI mortality rates in this cohort (15.6\% at 30 days and $22.5 \%$ at 1 year), there was no suggestion that CKD patients were at higher risk of short- or long-term mortality when treated with either a primary rate or rhythm control strategy. Interestingly, regardless of CKD status, there was no significant overall impact of a rhythm control strategy on discharge rhythm in this population. This finding does not rule out a possible benefit of rhythm control in reducing the overall duration and burden of AF during the hospitalization or improving quality of life and/or functional status, although this was not specifically addressed in our analysis. Results of the subgroup with moderate to severe CKD suggested that a rhythm control strategy was associated with a lower probability of maintaining SR at discharge. While results of any subgroup should be interpreted with caution, this is an intriguing finding that should be the subject of future prospective studies on antiarrhythmic management in CKD patients.

\section{Limitations of the study}

As with any observational study, the present one is not without some limitations. First, although this was a pre-specified prospective analysis of $\mathrm{AF}$ management and outcomes, treatment with rate vs. rhythm control was not randomized and the potential for confounding exists. This is mitigated somewhat by the multivariable regression modeling to adjust for known confounders in the cohort. Second, while in-hospital management was recorded and accounted for in the analysis, the impact of interventions after hospital discharge on 1 -year mortality, such as additional medications, or cross-over from rate control to a rhythm control strategy, was not known. Also, creatinine clearance 
was calculated using serum creatinine measurements at the time of study enrollment but this may not accurately reflect steady-state renal function. Finally, amiodarone and procainamide were the principal antiarrhythmic medications used in GUSTO-III and thus results in this cohort may not be generalizable to contemporary practice, where agents such as dofetilide, sotalol, and dronedarone are now commonly used in the post-MI setting.

\section{Conclusions}

In patients with post-MI AF, treatment with a rhythm or rate control strategy does not significantly impact short-term or long-term mortality, regardless of kidney disease status. Future randomized trials to investigate the optimal management of post-MI AF in patients with CKD are needed.

Conflict of interest: E.S.W. received research funding from HealthCare21; V.P.T. and K.E.C. have no disclosures; J.H.A. received research funding from: Bristol Myers Squibb, CSL, Daiichi Sankyo, Duke Health System, Medtronic Japan, Merck-Schering Plough, NIH, Pfizer, Regado Biosciences, and consulting fees from: Bayer, Boehringer Ingelheim, Bristol Myers Squibb, CSL, Daiichi Sankyo, Duke Health System, Duke Private Diagnostic Clinic, Ortho-McNeil-Jannsen, Polymedix, Pfizer, Regado Biosciences; H.D.W. received research funding from: Sanofi-Aventis, Eli Lilly, Medicines Company, National Institutes of Health, Pfizer, Roche, Johnson \& Johnson, Schering Plough, Merck Sharpe \& Dohme, AstraZeneca, GSK, Daiichi Sankyo Pharma Development, Bristol Myers Squibb, and has served on advisory boards for: Merck Sharpe \& Dohme, Regado Biosciences; E.M.O. received research funding from: Daiichi Sankyo, Datascope, Eli Lilly, and consulting fees from: AstraZeneca, Boehringer Ingelheim, Gilead Sciences, Liposcience, Merck, Sanofi-Aventis, The Medicines Company, WebMD, Pozen Inc; S.M.A. received research funding from Bristol Myers Squibb.

\section{References}

1. Reinecke H, Brand E, Mesters R et al. Dilemmas in the management of atrial fibrillation in chronic kidney disease. J Am Soc Nephrol, 2009; 20: 705-711.

2. Soliman EZ, Prineas RJ, Go AS et al. Chronic kidney disease and prevalent atrial fibrillation: The Chronic Renal Insufficiency Cohort (CRIC). Am Heart J, 2010; 159: 1102-1107.

3. McManus DD, Corteville DC, Shlipak MG et al. Relation of kidney function and albuminuria with atrial fibrillation (from the Heart and Soul Study). Am J Cardiol, 2009; 104: 1551-1555.

4. Wong CK, White HD, Wilcox RG et al. New atrial fibrillation after acute myocardial infarction independently predicts death: The GUSTO-III experience. Am Heart J, 2000; 140: 878-885.
5. Nilsson KR, Jr., Al-Khatib SM, Zhou Y et al. Atrial fibrillation management strategies and early mortality after myocardial infarction: results from the Valsartan in Acute Myocardial Infarction (VALIANT) Trial. Heart, 2010; 96: 838-842.

6. Wong CK, White HD, Wilcox RG et al. Management and outcome of patients with atrial fibrillation during acute myocardial infarction: The GUSTO-III experience. Global use of strategies to open occluded coronary arteries. Heart, 2002; 88: 357-362.

7. Smith GL, Masoudi FA, Shlipak MG et al. Renal impairment predicts long-term mortality risk after acute myocardial infarction. J Am Soc Nephrol, 2008; 19:141-150.

8. Shlipak MG, Heidenreich PA, Noguchi $\mathrm{H}$ et al. Association of renal insufficiency with treatment and outcomes after myocardial infarction in elderly patients. Ann Intern Med, 2002; 137: $555-562$.

9. A comparison of reteplase with alteplase for acute myocardial infarction. The Global Use of Strategies to Open Occluded Coronary Arteries (GUSTO III) Investigators. N Engl J Med, 1997; 337: 1118-1123.

10. Crenshaw BS, Ward SR, Granger $\mathrm{CB}$ et al. Atrial fibrillation in the setting of acute myocardial infarction: The GUSTO-I experience. Global Utilization of Streptokinase and TPA for Occluded Coronary Arteries. J Am Coll Cardiol, 1997; 30: 406-413.

11. Kober L, Swedberg K, McMurray JJ et al. Previously known and newly diagnosed atrial fibrillation: a major risk indicator after a myocardial infarction complicated by heart failure or left ventricular dysfunction. Eur J Heart Fail, 2006; 8: 591-598.

12. Al-Khatib SM, Pieper KS, Lee KL et al. Atrial fibrillation and mortality among patients with acute coronary syndromes without ST-segment elevation: results from the PURSUIT trial. Am J Cardiol, 2001; 88: A7, 76-79.

13. Pedersen OD, Abildstrom SZ, Ottesen MM et al. Increased risk of sudden and non-sudden cardiovascular death in patients with atrial fibrillation/flutter following acute myocardial infarction. Eur Heart J, 2006; 27: 290-295.

14. Julian DG, Camm AJ, Frangin G et al. Randomised trial of effect of amiodarone on mortality in patients with left-ventricular dysfunction after recent myocardial infarction: EMIAT. European Myocardial Infarct Amiodarone Trial Investigators. Lancet, 1997; 349: 667-674.

15. Pedersen OD, Bagger H, Kober L et al. The occurrence and prognostic significance of atrial fibrillation/-flutter following acute myocardial infarction. TRACE Study group. TRAndolapril Cardiac Evalution. Eur Heart J, 1999; 20: 748-754.

16. Watanabe H, Watanabe T, Sasaki S et al. Close bidirectional relationship between chronic kidney disease and atrial fibrillation: The Niigata preventive medicine study. Am Heart J, 2009; 158: 629-636.

17. Baber U, Howard VJ, Halperin JL et al. Association of Chronic Kidney Disease With Atrial Fibrillation Among Adults in the United States: REasons for Geographic and Racial Differences in Stroke (REGARDS) Study. Circ Arrhythm Electrophysiol, 2011; 4: 26-32.

18. Al Suwaidi J, Reddan DN, Williams K et al. Prognostic implications of abnormalities in renal function in patients with acute coronary syndromes. Circulation, 2002; 106: 974-980.

19. Gibson CM, Dumaine RL, Gelfand EV et al. Association of glomerular filtration rate on presentation with subsequent mortality in non-ST-segment elevation acute coronary syndrome; observations in 13,307 patients in five TIMI trials. Eur Heart J, 2004; 25 : 1998-2005.

20. Rodrigues FB, Bruetto RG, Torres US et al. Effect of kidney disease on acute coronary syndrome. Clin J Am Soc Nephrol, 2010; 5: 1530-1536.

21. Denetclaw TH, Oshima N, Dowling TC. Dofetilide dose calculation errors in elderly associated with use of the modification of diet in renal disease equation. Ann Pharmacother, 2011; 45: e44.

22. Patane S, Marte F, Di Bella G et al. QT interval prolongation, torsade de pointes and renal disease. Int J Cardiol, 2008; 130: e71-e73.

23. Kestenbaum B, Rudser KD, Shlipak MG et al. Kidney function, electrocardiographic findings, and cardiovascular events among older adults. Clin J Am Soc Nephrol, 2007; 2: 501-508. 